\title{
FACTORIZATION ALONG NEST ALGEBRAS
}

\author{
AVRAHAM FEINTUCH
}

\begin{abstract}
ABSTRACr. Let $T$ be a positive definite operator on $\mathcal{K}$ and $\mathscr{R}$ a nest algebra in $B(\mathcal{H})$. A necessary and sufficient condition is given for the existence of a factorization for $T$ of the form $T=A^{*} A$ with $A, A^{-1} \in \mathscr{R}$.
\end{abstract}

1. Introduction. While the theory of nest algebras began formally with the paper of Ringrose, the problem of factoring an operator with respect to a chain of subspaces of a Hilbert space is much older. In its simplest form the question was: can one factor a square matrix into the product of an upper triangular and lower triangular matrix? An infinite dimensional form of this equation was studied in the theory of Toeplitz matrices and in the solution of the discrete time Wiener-Hopf equation. The same type of problem with respect to a continuous chain arises in the solution of the standard Wiener-Hopf equation. These problems are very strongly related to optimal control problems for linear time invariant systems [3]. The problem we consider here is of the same flavour. If $\mathscr{N}$ is a nest of subspaces of a Hilbert space $\mathcal{H}$ and $T$ is a positive definite operator on $\mathcal{H}$, can $T$ be factored as $T=A^{*} A$ where $A, A^{-1} \in \operatorname{Alg} \mathfrak{K}$ ? This problem was solved for the case where $\mathfrak{\Re}$ is order isomorphic to the integers by Arveson [1], for the case where $T=I+V$ with $V$ Hilbert-Schmidt by Gohberg-Krein [2]. Recently, Larson [4] gave an example of a positive definite operator $T$ on $L^{2}[0,1]$ for which such a factorization does not exist with respect to the chain $\left\{L^{2}[0, t]: 0<t<1\right\}$. Here we give a necessary and sufficient condition for such a factorization to exist. This condition will be in terms of $T$ and $\Re$.

2. The main result. The first lemma is implicit in [1].

LEMMA 1. Given the family of projections $\left\{E_{N}: N \in \Re\right\}$, suppose there exists a unitary operator $U$ : for each $N \in \mathfrak{N}, U E_{N}=E_{T^{1 / 2} N} U$. Then $T$ has a factorization $A^{*} A$ where $A, A^{-1} \in \mathrm{Alg} \Re$.

Proof. Let $A=U^{*} T^{1 / 2}$. Then $A^{*} A=T$ and

$$
\begin{aligned}
A N & =A E_{N} \mathcal{H}=U^{*} T^{1 / 2} E_{N} \mathcal{H}=U^{*} E_{T^{1 / 2} N} \mathcal{H} \\
& =E_{N} U^{*} \mathcal{H}=E_{N} \mathcal{H}=N .
\end{aligned}
$$

$A N=A^{-1} N=N$ and the proof is complete.

Received by the editors January 3, 1981.

1980 Mathematics Subject Classification. Primary 47A68, 47C99.

(c) 1982 American Mathematical Society 0002-9939/82/0000-0408/\$01.75 
LEMMA 2. Let $B$ be a positive definite operator on $\mathcal{H}$. Define an (equivalent) inner product $[$,$] on \mathcal{H}$ by

$$
[x, y]=(B x, y) \text {. }
$$

Suppose $\mathfrak{T}$ is a subspace of $\mathcal{H}$ with associated orthogonal projection $P$. Then $\tilde{P}=(P B P+Q)^{-1} P B$ is the orthogonal projection on $\mathfrak{N}$ with respect to [ , ] $(Q=I-P)$.

Proof. Since $\tilde{P}$ is idempotent, $\tilde{P}^{2}=\tilde{P}$. A straightforward computation shows that $\tilde{P}$ is selfadjoint with respect to [ , ] if and only if $B \tilde{P}=\tilde{P} B^{*}$. Since $P$ and $\tilde{P}$ are idempotents with the same range, $P \tilde{P}=\tilde{P}, \tilde{P} P=P$ and $Q \tilde{P}=0$. Also, by taking adjoints, $P=P \tilde{P}^{*}$. Therefore,

$$
P B \tilde{P}=P \tilde{P}^{*} B=P B \text {. }
$$

But $P B \tilde{P}=P B P \tilde{P}=[P B P+Q] \tilde{P}$, and therefore $(P B P+Q) \tilde{P}=P B$. Since $B$ is positive definite, $P B P+Q$ is invertible and $\tilde{P}=[P B P+Q]^{-1} P B$. This completes the proof.

COROLlaRY 3. The orthogonal projection on $T^{1 / 2} E_{N} \mathcal{H}$ is $T^{1 / 2}\left[E_{N} T \mid E_{N}\right]^{-1} T^{1 / 2}$.

Proof. It is easily seen that the orthogonal projection on $T^{1 / 2} E_{N} \mathcal{H}$ with respect to the inner product, defined by $[x, y]=\left(T^{-1} x, y\right)$, is $T^{1 / 2} E_{N} T^{-1 / 2}$. Thus the projection in the standard inner product $(x, y)=[T x, y]$ will be

$$
\left[T^{1 / 2} E_{N} T^{-1 / 2} T T^{1 / 2} E_{N} T^{-1}+\left(I-T^{1 / 2} E_{N} T^{-1 / 2}\right)\right]^{-1} T^{1 / 2} E_{N} T^{1 / 2} T,
$$

which when simplified gives $T^{1 / 2}\left[E_{N} T \mid E_{N} \mathcal{H}\right]^{-1} T^{1 / 2}$.

TheOREM 4. Suppose $T$ is a positive definite operator on $\mathcal{H}$. Then $T=A^{*} A$ with $A, A^{-1} \in \mathrm{Alg} \mathcal{X}$ if and only if there exists a unitary operator $U$ such that

$$
U E_{N}=T^{1 / 2}\left[E_{N} T \mid E_{N} \mathcal{H}\right]^{-1} T^{1 / 2} U
$$

for all $N \in \mathcal{H}$.

Proof. If such a $U$ exists, we have seen that $T$ has the required factorization. Now suppose $T=A^{*} A$ with $A, A^{-1} \in \operatorname{Alg} \Re$. Then

$$
\begin{aligned}
T^{1 / 2}\left[E_{n} T \mid E_{N} \mathcal{H}\right]^{-1} T^{1 / 2} & =\left[E_{N} A^{*} A \mid E_{N} \mathcal{H}\right]^{-1} T^{1 / 2} \\
& =T^{1 / 2}\left[E_{N} A^{*} E_{N} A E_{N} \mid E_{N} \mathcal{H}\right]^{-1} T^{1 / 2} .
\end{aligned}
$$

Since $A^{-1} \in \mathrm{Alg} \mathscr{T}$, it is easily seen that

$$
\begin{aligned}
{\left[E_{N} A^{*} E_{N} A E_{N} \mid E_{N} \mathcal{H}\right]^{-1} } & =\left[E_{N} A E_{N} \mid E_{N} \mathcal{H}\right]^{-1}\left[E_{N} A^{*} E_{N} \mid E_{N} \mathcal{H}\right]^{-1} \\
& =\left[E_{N} A^{-1} E_{N} \mid E_{N} \mathcal{H}\right]\left[E_{N} A^{*-1} E_{N} \mid E_{N} \mathcal{H}\right] .
\end{aligned}
$$

Thus $T^{1 / 2}\left[E_{N} T \mid E_{N} \mathcal{H}\right]^{-1} T^{1 / 2}=T^{1 / 2}\left[E_{N} A^{-1} E_{N} \mid E_{N} \mathcal{H}\right]$.

$$
\begin{aligned}
{\left[E_{N} A^{*-1} E_{N} \mid E_{N} \mathcal{H}\right] } & T^{1 / 2}=T^{-1 / 2} T\left[E_{N} A^{-1} E_{N} \mid E_{N} \mathcal{H C}\right]\left[E_{N} A^{*-1} E_{N} \mid E_{N} \mathcal{H C}\right] T T^{-1 / 2} \\
= & T^{1 / 2} A^{*} A\left[E_{N} A^{-1} E_{N} \mid E_{N} \mathcal{H}\right]\left[E_{N} A^{*-1} E_{N} \mid E_{N} \mathcal{H}\right] T T^{-1 / 2} \\
= & T^{-1 / 2} A^{*}\left[E_{N} A E_{N} A^{-1} E_{N} \mid E_{N} \mathcal{H}\right]\left[E_{N} A^{*-1} E_{N} A^{*} E_{N} \mid E_{N} \mathcal{H}\right] A T^{-1 / 2} \\
= & T^{-1 / 2} A^{*} E_{N} A T^{1 / 2}
\end{aligned}
$$


Noting that $U=T^{-1 / 2} A^{*}$ is unitary, we obtain that $E_{T^{1 / 2} N} U=U E_{N}$ and the proof is complete.

\section{BIBLIOGRAPHY}

1. W. A. Arveson, Interpolation in nest algebras, J. Funct. Anal. 20 (1975), 208-233.

2. I. C. Gohberg and M. G. Krein, Theory and applications of Volterra operators in Hilbert space, Transl. Math. Monos., vol. 24, Amer. Math. Soc., Providence, R.I., 1970.

3. T. Kailath, Lectures on linear least-square estimation, Springer-Verlag, New York, 1976.

4. D. Larson (preprint).

Department of Mathematics, Ben-Gurion University, Beersheva, Israel 